\title{
Anatomy of the Aortic Root and the Coronary Ostia: CT Evaluation
}

\author{
Uliana Pidvalna, ${ }^{1}$ Lesya Mateshuk-Vatseba, ${ }^{1}$ Dmytro Beshley, ${ }^{2,3}$ Marta Kolishetska ${ }^{1}$ \\ 'Danylo Halytsky Lviv National Medical University \\ ${ }^{2}$ Ukrainian-Polish Heart Center Lviv \\ ${ }^{3}$ Lviv Regional Clinical Hospital
}

Disclose and conflicts of interest: none to be declared by all authors

\begin{abstract}
Introduction: as there is a growing number of percutaneous interventions, scrupulous attention and profound knowledge of the aortic root and neighboring structures is required. The objective of the study is to describe the anatomy of the aortic root and the coronary ostia in asymptomatic patients using CT.

Methods: eighty-five patients without any clinical symptoms suggestive of the aortic root and coronary arteries diseases underwent contrast-enhanced MDCT. CT angiography was performed with a 64-row helical CT scanner after administration of the non-ionic, iodinated contrast agent.

Results: CT angiography depicted the entire structure of the aortic root. The aortic root encompasses several distinct elements, namely the aortic annulus, the aortic valve leaflets and their (leaflet) attachments, the interleaflet triangles, the sinuses of Valsalva, the coronary arteries ostia and the sinotubular junction. CT angiography depicted the distance between the aortic annulus and the margins of the left and right coronary ostia. While carrying out the analysis, the precise description of the anatomy of the sinotubular junction and the valve leaflet attachments has to be made. Sinuses of Valsalva normally consist of the right, left and posterior cusps. Left and right coronary ostia are located below the upper margin of the cusps.

Conclusion: When analyzing the anatomy of the aortic root and the coronary ostia, each entity should be examined separately. It is important to take into account the relationship between the structures the aortic root encompasses. Changes in the size of one structure cause the improper functioning of the adjacent ones.
\end{abstract}

Keywords: Anatomy; Aorta; Coronary arteries; Sinuses of Valsalva; Computed tomography.

\section{Introduction}

The mortality rate from cardiovascular diseases (CVDs) ranks first among all the diseases globally. According to the World Health Organization, in 2016 about 17.9 million people died of CVDs (15.2 million of whom died of heart attack and stroke). ${ }^{1}$ This worrying trend is urging us to improve the diagnostics and treatment of CVDs. Recent decades have been marked by the technological advances and introduction of innovative techniques in cardiology and cardiac surgery. ${ }^{2}$ The increasing number of such percutaneous interventional procedures as coronary angiography, coronary angioplasty and stenting, intravascular ultrasound (IVUS) and fractional flow reserve (FFR), transcatheter aortic valve implantation (TAVI), requires detailed knowledge of the anatomy of the aortic root. Preoperative planning is an important factor in the successful performance of cardiovascular procedures. The geometry of the aortic root and adjacent structures has to be estimated. Careful attention needs to be paid to the height of the takeoff of the coronary artery which is measured from the aortic valve annulus. The diameter of the coronary ostium has to be considered as well. An error in the calculations can lead to the coronary ostium occlusion by a valve (in TAVI) or an inability to put an introducer into the coronary artery ostium. Non-invasive methods are the best option for the preoperative assessment of anatomy. ${ }^{3}$ Contrastenhanced multidetector computed tomography (MDCT) provides a detailed visualization of the anatomy of the studied structures, taking into account the spatial image. ${ }^{4}$

The study aims to describe the anatomy of the aortic root in patients without any structural damage of the heart and ascending aorta and to assess the morphological features of the coronary arteries, which arise from the sinuses of Valsalva with the help of MDCT.

\section{Material and Methods}

Study Design and Patient Selection. Patients of the Lviv Regional Clinical Hospital (Lviv, Ukraine) and Ukrainian-Polish heart center "Lviv" (Lviv, Ukraine) were involved in the given study.

Inclusion criteria were as follows:

1. chest CT with contrast enhancement

2. no clinical symptoms of heart disease and ascending aorta disease

3. no CT signs of structural heart disease and ascending aorta disease 
4. the signing of the informed consent on the use of data in the study by the patient or official representative (according to the Declaration of Helsinki and the national legal regulations (Ethics Committee Approval: Bioethics Commission of Danylo Halytsky Lviv National Medical University, protocol No. 423 April 2018)).

CT images of 86 patients were obtained with the following gender distribution: 48 men and 38 women. The average age of patients was $53.8 \pm 15.3$ years. The examinations were conducted between September 2020 and January 2021.

The patients were scanned on MDCT scanner LightSpeed VCT XT, GE (General Electric, USA) with 64-row chest CT after administration of nonionic, iodinated contrast agent. Scan type - Helical. Intravenous contrast material - 80-110 mL of Ultravist 470 (Bayer Healthcare, Germany) and 30-40 mL of saline (Arterium, Ukraine) at $4 \mathrm{~mL} / \mathrm{s}$. A standard technique protocol was used according to the manufacture guidelines. $\mathrm{kV}$ and $\mathrm{mAs} /$ rotation were variables based on the patient's size. Additional parameters: rotation time $0.33 \mathrm{~s}$; dual-source $64 \times 0.6$ collimation; automated patient instruction on inspiration; heart rate $60-70$ beats/min.

Data analysis. CT images were analyzed on a dedicated CT workstation (General Electric, USA) with all the necessary software. The study is based on CT images of the aortic annulus, sinuses of Valsalva and sinotubular junction; the distance between aortic annulus to the separate inferior and superior margins of both left coronary artery (LCA) and right coronary artery (RCA); the distance between aortic annulus and sinotubular junction. Values are presented in mean \pm standard deviation.

\section{Results}

The aortic root is a complex anatomical structure that consists of multiple entities. It encompasses the aortic valve annulus, the aortic valve leaflets, the leaflet attachments and the interleaflet triangles, the sinuses of Valsalva, the coronary ostia and the sinotubular junction. Any changes in the geometry of the aortic root structures can lead to the structural aortic valve deformations, dilatation of the sinotubular junction and the sinuses of Valsalva. Therefore, a comprehensive assessment of all the entities is important.

Clear visualization of the studied structures can be obtained with the help of CT. The CT image consists of a matrix of pixels, each of which is recognized by a computer system ranging from -1000 to +1000 Hounsfield units (HU). HUs depend on the density of the tissue that has been scanned. It is known that air has -1000 HU, water $0 \mathrm{HU}$, soft tissues 20-100 HU, skeletal system 400-600 HU. Thus, by measuring the density we can differentiate the structure of tissues. Dense substances absorb more X-rays which results in higher HU. The images appear to be lighter and have increased attenuation.
According to the examination protocol, each patient was scanned without contrast enhancement and with it. CT examination without contrast enhancement made it possible to visualize structures with high density. Increased attenuation in the aortic valve indicates calcification of the aortic valve; in the area of the ascending aorta - calcification of the aortic intima. Patients who suffered from heart disease and ascending aorta disease were not included in the study.

Contrast-enhanced CT provided better visualization of all the components of the aortic root and the coronary ostia. Injection of contrast material into the blood vessels allows us to distinguish between the structures that are vascularized. The course of the vessels, the morphology of their lumen and walls can be studied as well. After the survey, the post-processing of digital images took place. It enabled us to carry out an additional detailed analysis.

CT images were analyzed in the horizontal (axial), sagittal or frontal (coronal) planes. Volumetric data were reconstructed into a 3D image. The image itself, because of its authenticity, is an excellent method of studying the anatomy of the heart and aorta.

Analysis of the contrast-enhanced CT chest images shows that the sinotubular junction (STJ) is the upper margin of the aortic root (Figure 1). This is the junction of the dilated part of the aorta, which contains the sinuses of Valsalva, with the narrowing of the ascending aorta. However, it is not a clearly defined circular ridge. The three commissions at the STJ level are viewed as an outpost of the leaflet attachments. The morphology of both STJ and commissures is considered while analyzing data. In the group of patients under

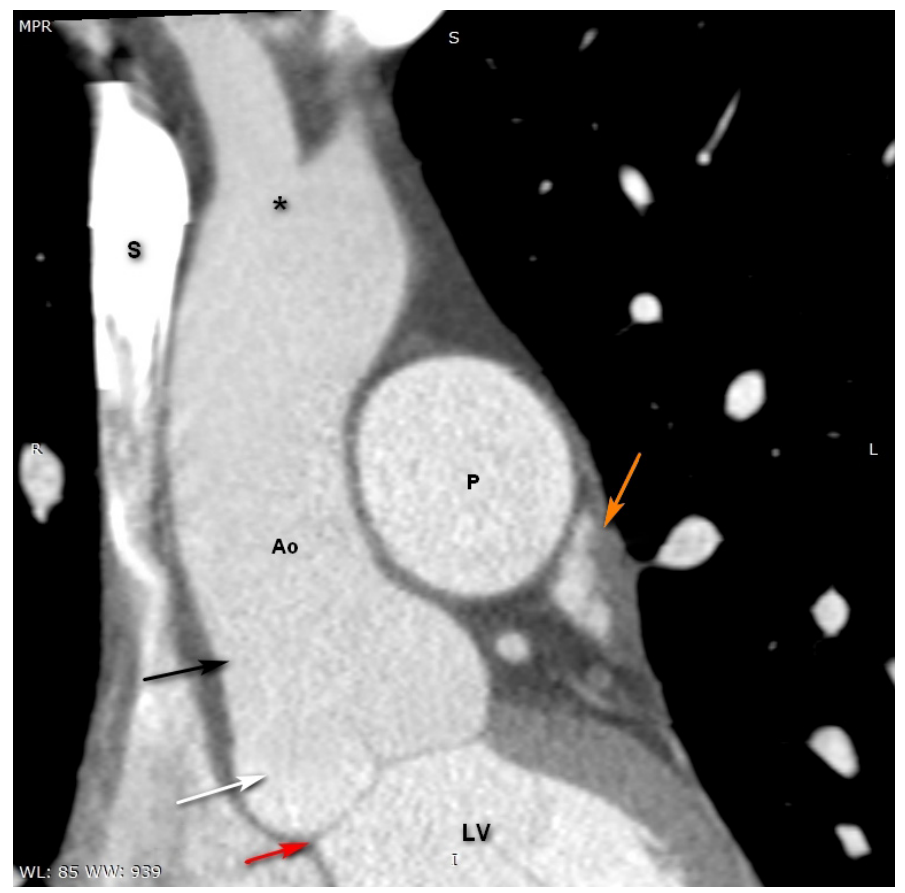

Figure 1. Coronal CT image shows the ascending aorta (Ao), aortic valve (red arrow), sinuses of Valsalva (white arrow), sinotubular junction (black arrow), left ventricle (LV), pulmonary artery (P), superior vena cava (S), the origin of the great vessels $(*)$, left atrial appendage (orange arrow). Contrast-enhanced CT 
examination, the structural proportions corresponded to the norm. Dilatation of the sinotubular junction can cause a weakening of the valve leaflet strain. Dilatation of STJ and intercommissural distance provokes inappropriate coaptation of the aortic valve. That is, it can conduct toward the aortic valve insufficiency.

Assessment of the anatomy of the sinotubular junction and valve leaflets (Figure 2) was performed on the short axis and oblique coronal view. Below the sinotubular junction, there are the sinuses of Valsalva (Figure 3). Normally, they consist of three cusps - left, right and posterior. The cusps have two surfaces: convex (lower) and concave (upper), resembling bags in shape. The convex surface faces the left ventricle (where the aorta originates), while concave directly faces the lumen of the aorta, forming the aortic sinus. During diastole, the aortic valve should close tightly to prevent backflow into the left ventricular cavity. This mechanism, involving many other factors, is also maintained by the anatomical peculiarities of the valve leaflets, namely nodules and lunula. Nodule of the semilunar leaflet (nodulus valvulae semilunaris) - "body of Arantius" - is a thickening of the valve in the middle of the free edge. Delicate folds spread from the nodule to the sides - they are called lunula (lunulae valvulae semilunaris). The semilunar cusps are attached to the aortic wall forming a relation to each other which is called commissure of semilunar valve cusps (commissurae valvulae semilunaris) and fibrous triangles between the sinuses. The triangle which is formed between the right and posterior cusps is clinically important, as it points out the location of the atrioventricular conduction bundle.

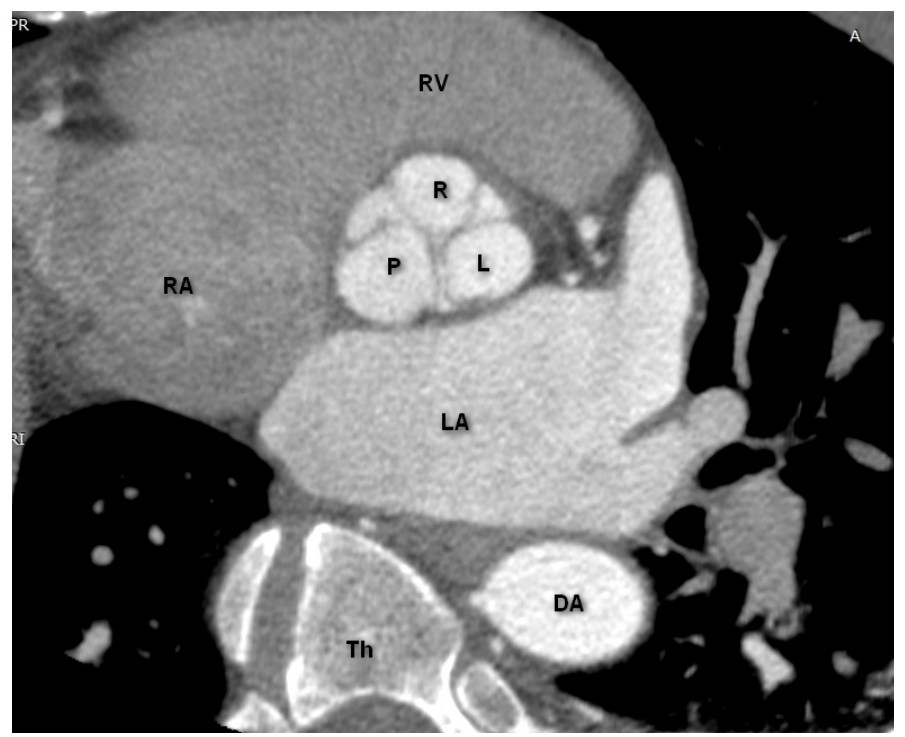

Figure 2. Oblique axial reformat through the aortic valve shows the normal anatomy of the aortic valve with complete coaptation of smooth-edged valve cusps. Right semilunar aortic valve (right coronary cusp, R), left semilunar aortic valve (left coronary cusp, L), posterior semilunar aortic valve (posterior coronary cusp, P), left atrium (LA), right ventricle (RV), right atrium (RA), descending aorta (DA), thoracic vertebra (Th). Contrast-enhanced CT
When assessing the aortic root, it is important to analyze the adjacent structures. The right and left coronary arteries originate from the sinuses of Valsalva (Figure 4). Normally, the coronary arteries originate above the corresponding sinuses of Valsalva. As the coronary arteries tend not to arise from the posterior sinus, it is also called the noncoronary sinus.

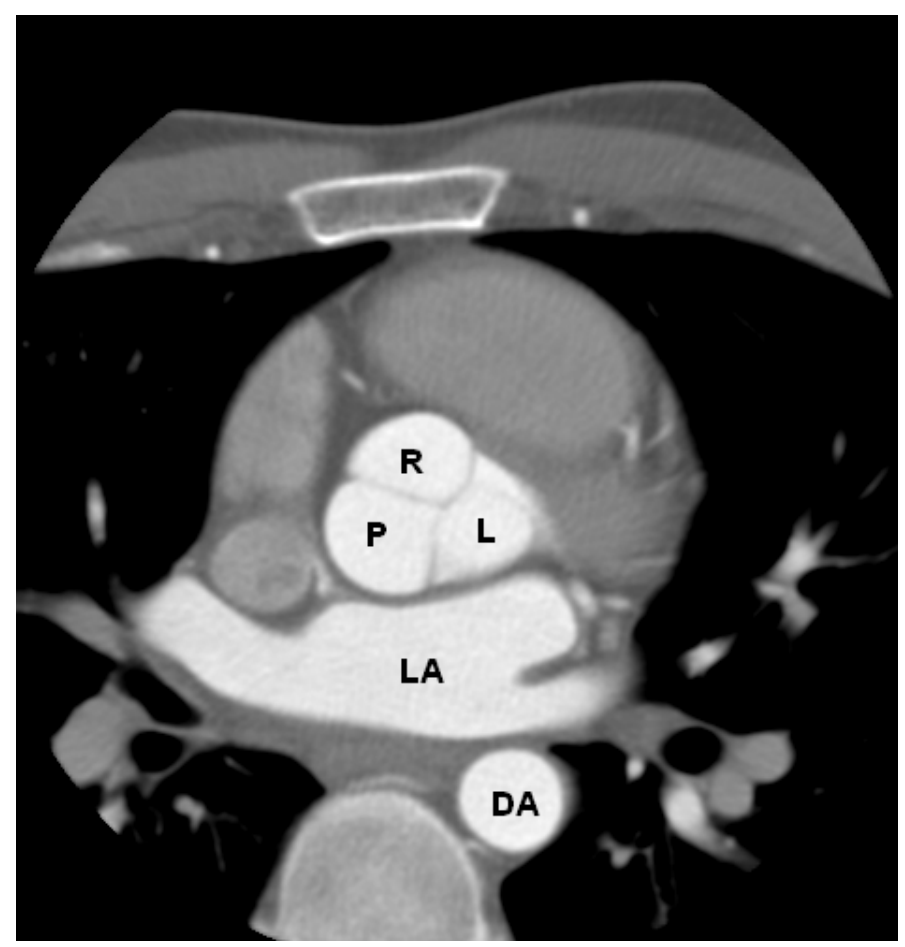

Figure 4. Oblique axial reformat through the aortic valve shows the normal anatomy of the sinuses of Valsalva. Right semilunar aortic valve (right coronary cusp, R), left semilunar aortic valve (left coronary cusp, L), posterior semilunar aortic valve (posterior coronary cusp, P), left atrium (LA), descending aorta (DA). Contrast-enhanced CT

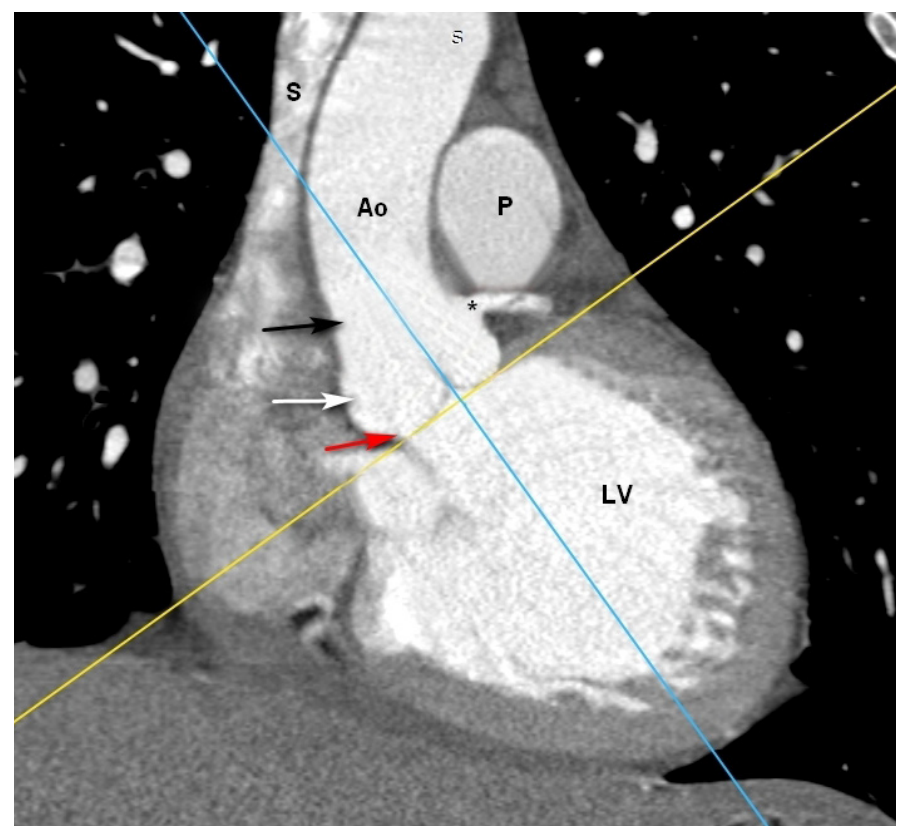

Figure 4. Coronal CT image shows the ascending aorta (Ao), aortic valve (red arrow), left ventricle (LV), pulmonary artery ( $P$ ), superior vena cava (S), the origin of the left coronary artery $\left(^{*}\right)$, sinuses of Valsalva (white arrow), sinotubular junction (black arrow). Contrast-enhanced CT 

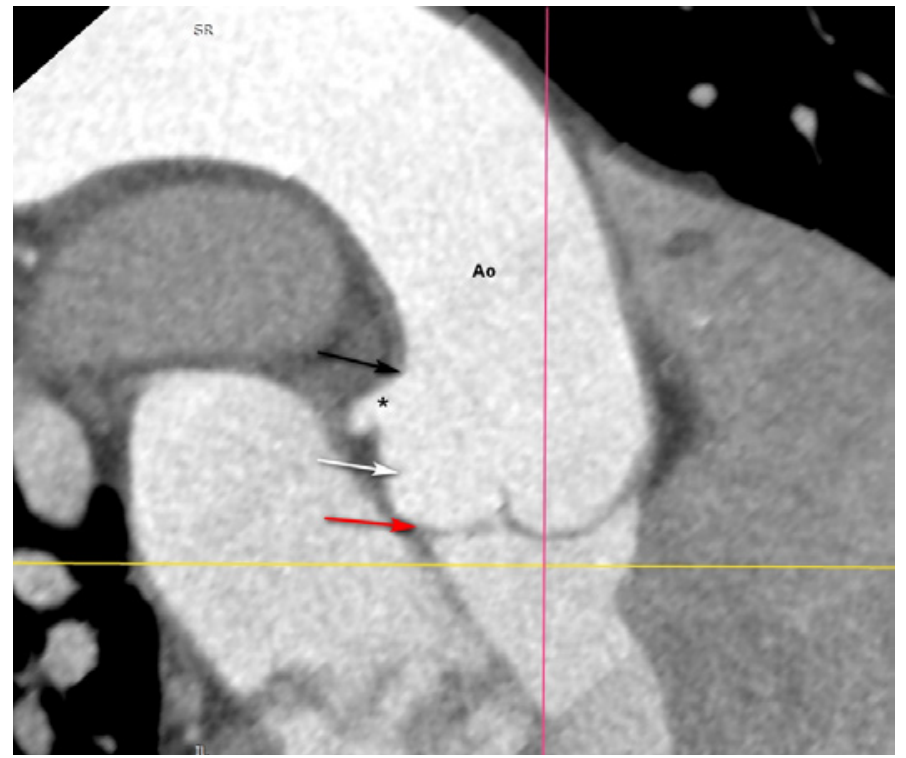

Figure 5. CT image shows the ascending aorta (Ao), the origin of the right coronary artery $\left({ }^{*}\right)$, aortic valve (red arrow), sinuses of Valsalva (white arrow), sinotubular junction (black arrow). Contrast-enhanced CT

In most cases, the coronary ostia are located under the upper edge of the aortic valve cusps. The height of the corresponding sinuses of Valsalva is larger than the height of the valves. Such a position allows the maximum blood supply to the coronary vessels in the left ventricular diastole. The coronary artery takeoff which is up to $5 \mathrm{~mm}$ above the sinotubular junction is also believed to be within the anatomical norm.

CT without contrast-enhancement makes it possible to determine the calcium index in the coronary vessels. The presence of calcium is an indicator of the damage to the intima caused by atherosclerosis. The patients with the given condition were not included in the study. Contrast-enhanced CT scans were used to visualize the location of the vessel and its course. No abnormalities in the origin or course of the coronary arteries were found in the examined patients. To assess the origin of the coronary arteries, it is mandatory to analyze images not only along the long and short axes but also oblique coronal.

The diameter of the coronary ostia (Figure 6, Figure 7 ) is determined on the horizontal (axial) sections. The height of the discharge of the coronary arteries is calculated as the distance from the aortic annulus (Figure 8) to the lower edge of the coronary artery ostium. Additionally, the measurements of the upper edge of the coronary ostium are taken. It was found out that the diameter of the left coronary artery is larger than the diameter of the right. Distally, the diameter of the vessels is gradually getting smaller.

If the coronary artery originates more than $1 \mathrm{~cm}$ above the sinotubular junction, it is referred to as "high takeoff". It might be associated with decreased coronary perfusion and myocardial ischemia. "Low coronary artery takeoff" is the location of the ostium less than $1 \mathrm{~cm}$ above the functional aortic valve annulus. If it is asymptomatic, it is not considered to be a critical abnormality. It is important to take it into account in cases of cardiac surgery, especially when the aortic valve is manipulated.

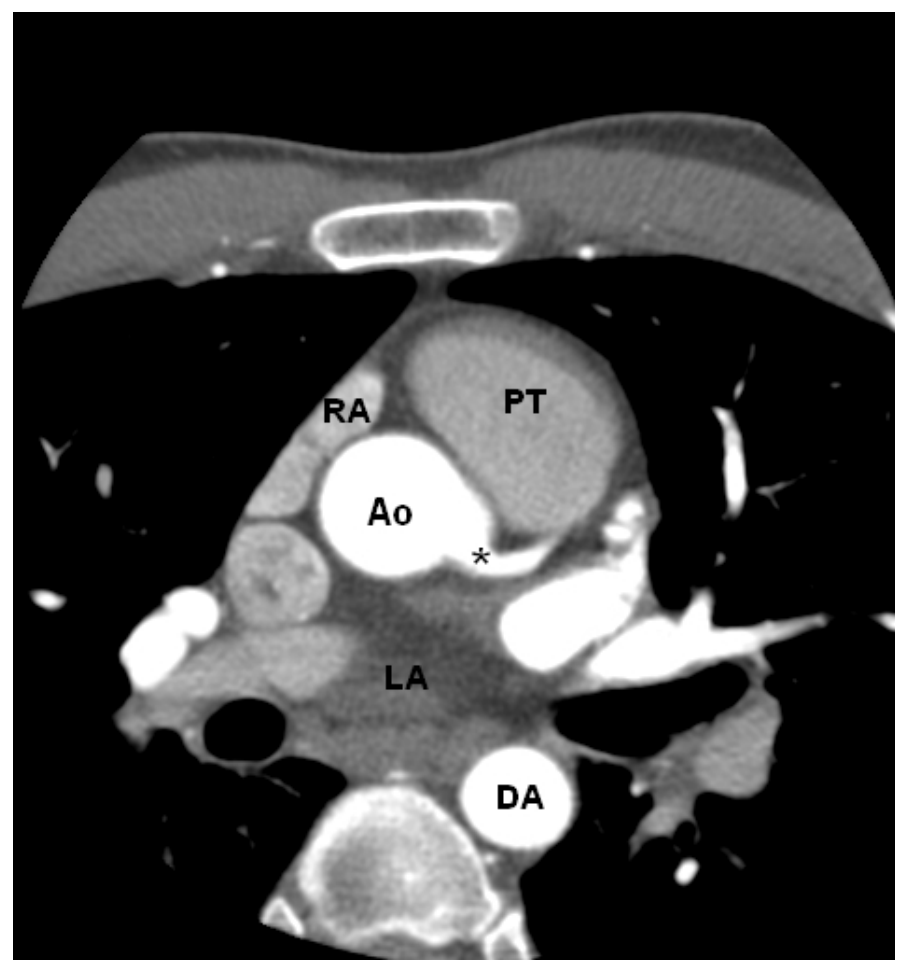

Figure 6. Oblique axial reformat through the aortic valve shows the origin of the left coronary artery $\left(^{*}\right)$. Aorta (Ao), left atrium (LA), right atrium (RA), pulmonary trunk (PT), descending aorta (DA). Contrast-enhanced CT

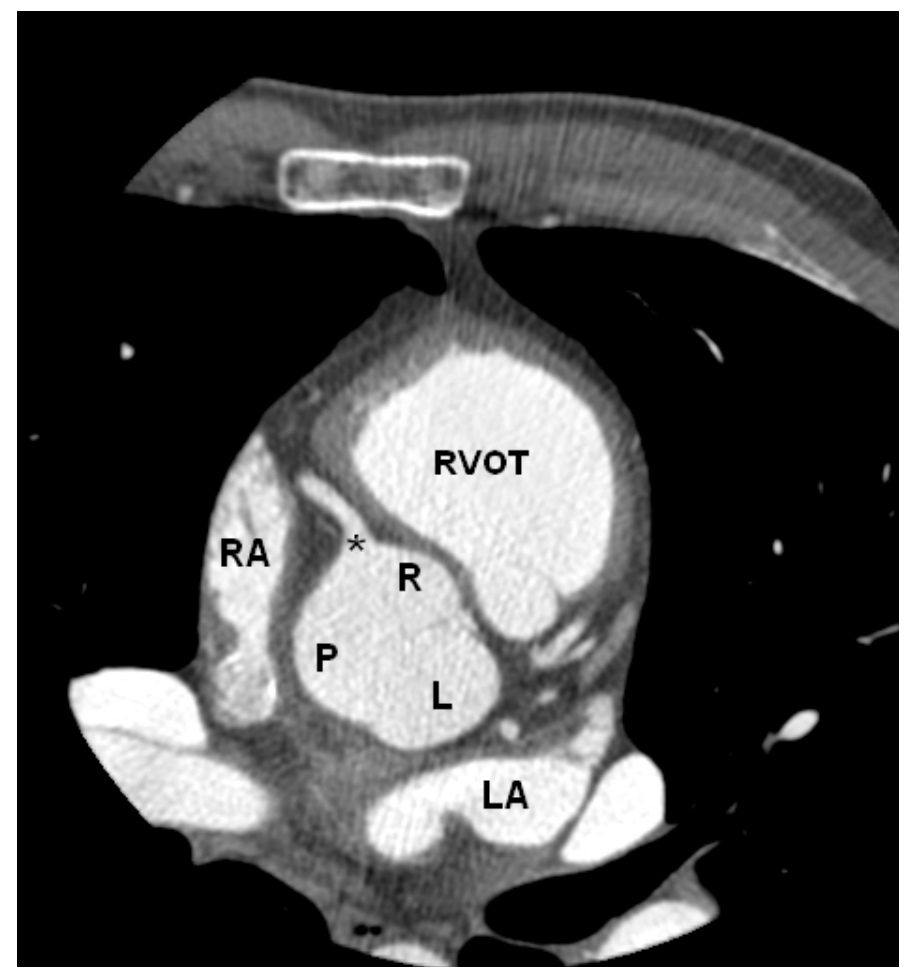

Figure 7. Oblique axial reformat through the aortic valve shows the normal anatomy of the aortic valve and the origin of the right coronary artery (*). Right semilunar aortic valve (right coronary cusp, R), left semilunar aortic valve (left coronary cusp, L), posterior semilunar aortic valve (posterior coronary cusp, $\mathrm{P}$ ), left atrium (LA), right atrium (RA), right ventricle outflow tract (RVOT), Contrastenhanced CT 


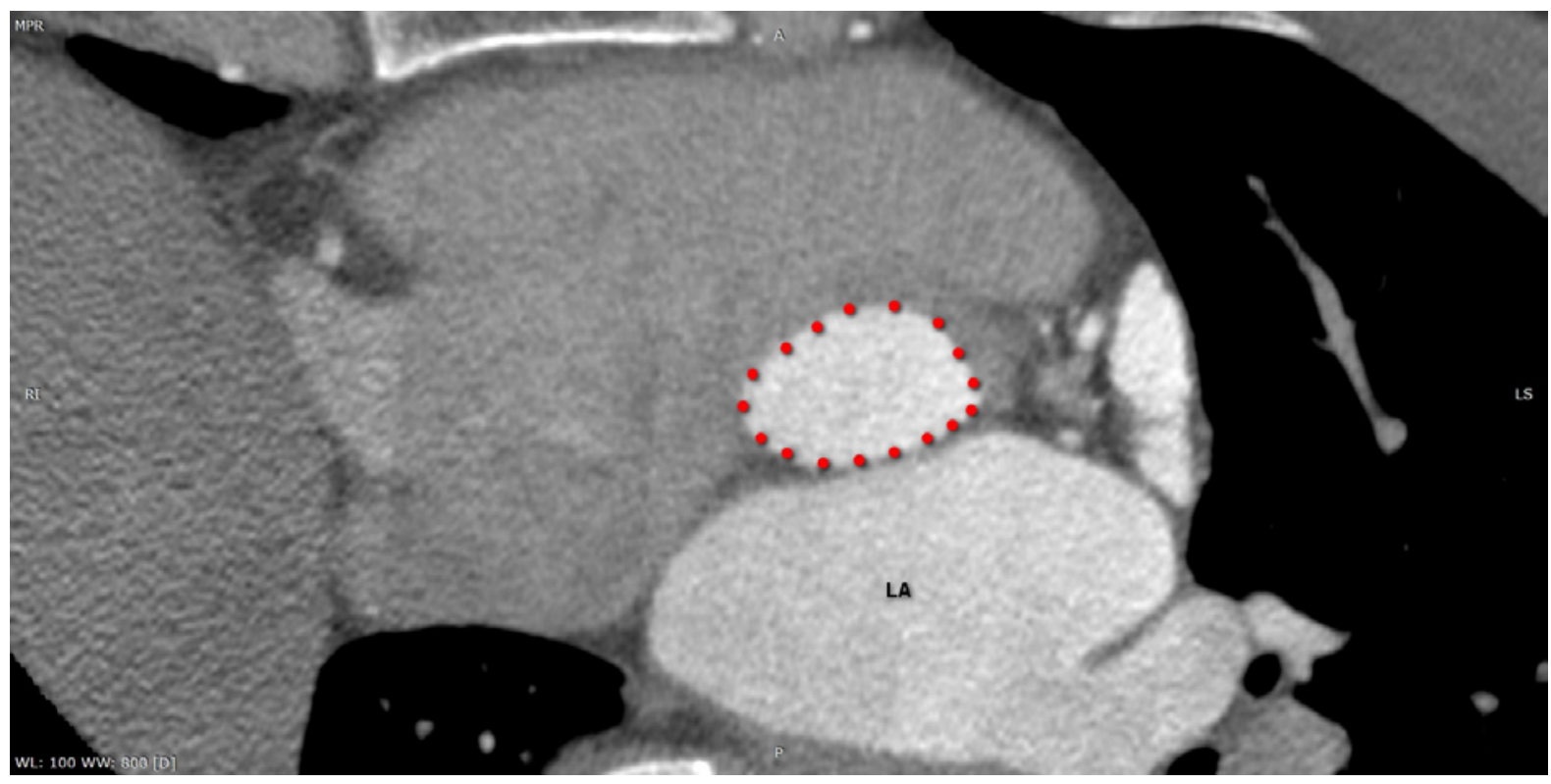

Figure 8. Axial (transverse) CT image of the thorax at the level of the aortic annulus (red dots). LA, left atrium. Contrast-enhanced CT

\section{Discussion}

The results of the given work are a fragment of the scientific research project "Morpho-functional features of organs in the pre- and postnatal periods of ontogenesis, under the influence of opioids, dietary supplements, reconstructive surgery and obesity" (State Registration Number 0120U002129.), which is being conducted at the Department of Normal Anatomy of Danylo Halytsky Lviv National Medical University.

The proper selection of patients was fundamental to the study since the aim was to analyze CT images of the patients without any aortic root and coronary vessels disease.

A variety of methods is available to diagnose the cardiovascular system. Ultrasound examination of the heart and ascending aorta, CT and Magnetic resonance imaging (MRI) $)^{5,6}$ are most often used to visualize the aortic root. Each method has its advantages and disadvantages, which have been described in detail by numerous authors, ${ }^{7-10}$ based on several number of studies and observations. We would like to focus on the rationale for the use of $\mathrm{CT}$ in the given study. Reliable measurements of the structures under study can be performed during transthoracic or transesophageal echocardiography. However, the distance from the aortic valve annulus to the left coronary artery ostium cannot be assessed. It is caused by the fact that the left coronary artery is not located in the standard plane in which the examination is performed. ${ }^{11} \mathrm{MRI}$ examination is time-consuming and is not always available. ${ }^{12} \mathrm{CT}$ angiography is the gold standard for evaluation of acute aortic pathology. According to data presented in numerous publications and recommendations,,${ }^{9,13-15}$ adequate assessment of the aortic anatomy can be performed using CT. CT scans provide a comprehensive assessment of the position of the coronary arteries in concerning the aortic annulus and the valve leaflets.

The aortic root is a complex structure, which consists of many elements. Adequate functioning of the cardiovascular system is made possible due to the coordinated work of the components of the aortic valve, maintenance of the size of the sinuses of Valsalva and sinotubular junction. ${ }^{16-17}$ It should be noted that it is still a controversial issue whether to consider the aortic root a part of the ascending aorta ${ }^{18}$ or a separate part. $^{7}$

When assessing the aortic root, the coronary ostia should be primarily focused on. It is crucial before planning cardiac procedures or cardiac surgery. ${ }^{2,11,19-21}$ Normally, the coronary arteries originate from the sinuses of Valsalva, below the STJ. "Low takeoff", which might be asymptomatic, is the location of the ostia less than $1 \mathrm{~cm}$ above the functional aortic valve annulus. ${ }^{22}$ "High takeoff" can be a potentially threatening condition, as the coronary artery originates more than $1 \mathrm{~cm}$ above the sinotubular junction. ${ }^{23}$ The results of the assessment of the diameter of the coronary arteries are consistent with the published data. Interestingly, with age, particularly in people over 75 , the diameter of the right coronary artery is slightly bigger than the left. ${ }^{24}$ Distally, the diameter of the vessel is gradually getting smaller, branching to the arterioles and capillaries. ${ }^{25}$

\section{Conclusion}

When analyzing the anatomy of the aortic root and the coronary ostia, each entity should be examined separately. It is important to take into account the relationship between the structures the aortic root encompasses. Changes in the size of one structure cause the improper functioning of the adjacent ones. 


\section{References}

1. World Health Organization. Cardiovascular diseases [Internet]. 2017. Available at: https://www.who.int/news-room/fact-sheets/ detail/cardiovascular-diseases-(cvds). Accessed Fevruary 3, 2021. 2. Beshley D, Dudek D, Wojdyla R, Pidvalna U, Averchuk V, Kulyk L. Innovative cardiology and cardiac surgery in Lviv. Proc Shevchenko Sci Soc Med Sci 2020;62(2):143-149.

3. Hennessey B, Vera-Urquiza R, Mejía-Rentería H, Gonzalo N, Escaned J. Contemporary use of coronary computed tomography angiography in the planning of percutaneous coronary intervention. Int J Cardiovasc Imaging [Internet]. 2020;36(12). Available at: https://pubmed.ncbi.nlm.nih.gov/33201348/. Accessed March 4, 2021.

4. Akhtar M, Tuzcu EM, Kapadia SR, Svensson LG, Greenberg RK, Roselli EE, et al. Aortic root morphology in patients undergoing percutaneous aortic valve replacement: Evidence of aortic root remodelling. J Thorac Cardiovasc Surg. 2009;137(4):950-956.

5. Yusuf Beebeejaun M, Malec A, Gupta R, Alkhawam H. Conservative management of chronic aortic dissection with underlying aortic aneurysm. Heart Int [Internet]. 2013;15;8(1):e4-e4. Available at: https://www.ncbi.nlm.nih.gov/pubmed/24179638. Accessed February 14, 2021.

6. Molossi S, Agrawal H, Mery CM, Krishnamurthy R, Masand $P$, Sexson Tejtel SK, et al. Outcomes in Anomalous Aortic Origin of a Coronary Artery Following a Prospective Standardized Approach. Circ Cardiovasc Interv. 2020;13(2):e008445.

7. Freeman LA, Young PM, Foley TA, Williamson EE, Bruce CJ, Greason $\mathrm{KL}$. CT and MRI assessment of the aortic root and ascending aorta. Am J Roentgenol. 2013;200(6):581-92.

8. Elsayed RS, Cohen RG, Fleischman F, Bowdish ME, Gawinecka J, Schönrath $F$, et al. Acute Type A Aortic Dissection. Am J Roentgenol [Internet]. 2017;35(6):90033. Available at: http://dx.doi. org/10.1016/j.ccl.2017.03.004. Accessed February 12, 2021.

9. Loewe C. Acute Aortic Syndromes. In: Multislice CT. Springer International Publishing AG; 2019:825-53.

10. Gawinecka J, Schönrath F, von Eckardstein A. Acute aortic dissection: pathogenesis, risk factors and diagnosis. Swiss Med Wkly. 2017;147:w14489.

11. Bleakley C, Eskandari M, Monaghan M. 3D transoesophageal echocardiography in the TAVI sizing arena: should we do it and how do we do it? Echo Res Pract. 2017;4(1):R21-R32.

12. Jones PA, Wilson JS. The Potential for Quantifying Regional Distributions of Radial and Shear Strain in the Thoracic and Abdominal Aortic Wall Using Spiral Cine DENSE MRI. J Biomech Eng. [Internet]. 2021 Feb 4; Available at: https://pubmed.ncbi.nlm.nih. gov/33537707/. Accessed March 4, 2021.

13. Wang S-W, Huang Y-B, Huang J-W, Chiu C-C, Lai W-T, Chen C-Y. Epidemiology, Clinical Features, and Prescribing Patterns of
Aortic Aneurysm in Asian Population From 2005 to 2011. Medicine. 2015;94(41):e1716-e1716.

14. Task A, Erbel R, Germany C, Aboyans V, France C, France CB, et al. 2014 ESC Guidelines on the diagnosis and treatment of aortic diseases Document covering acute and chronic aortic diseases of the thoracic The Task Force for the Diagnosis and Treatment of Aortic Diseases of the European Society of Cardiology (ESC). Eur Heart J. 2014;(35):2873-926.

15. Goldstein SA, Evangelista A, Abbara S, Arai A, Asch FM, Badano LP, et al. Multimodality imaging of diseases of the thoracic aorta in adults: From the American society of echocardiography and the European association of cardiovascular imaging: Endorsed by the society of cardiovascular computed tomography and society for cardiova. J Am Soc Echocardiogr. 2015;28(2):119-82.

16. Ho SY. Structure and anatomy of the aortic root. Eur J Echocardiogr. 2009;10(1):i3-10.

17. Loukas M, Bilinsky E, Bilinsky S, Blaak C, Tubbs RS, Anderson RH. The anatomy of the aortic root. Clin Anat. 2014;27(5):748-56.

18. Ho SY. Structure and anatomy of the aortic root. Eur J Echocardiogr. 2009;10(1):3-10.

19. Mori S, Tretter JT, Toba T, Izawa Y, Tahara N, Nishii T, et al. Relationship between the membranous septum and the virtual basal ring of the aortic root in candidates for transcatheter implantation of the aortic valve. Clin Anat. 2018;31(4):525-34.

20. Figulla HR, Franz M, Lauten A. The History of Transcatheter Aortic Valve Implantation (TAVI)-A Personal View Over 25 Years of development. Cardiovasc Revascularization Med. 2020;21(3):398403.

21. Thiele H, Kurz T, Feistritzer H-J, Stachel G, Hartung P, Eitel I, et al. Comparison of newer generation self-expandable vs. balloonexpandable valves in transcatheter aortic valve implantation: the randomized SOLVE-TAVI trial. Eur Heart J. 2020;41(20):1890-9.

22. Withana M, Uribe C, Gregoric ID, Angelini P. Low Origin of the Coronary Arteries and a Small Aortic Annulus Complicating Aortic Valve Replacement. Texas Hear Inst J. 2019;46(3):222-224.

23. Waller BF, Orr CM, Slack JD, Pinkerton CA, Van Tassel J, Peters T. Anatomy, histology, and pathology of coronary arteries: A review relevant to new interventional and imaging techniques-Part II. Clin Cardiol. 1992;15(7):535-40.

24. Ambrose MB, Avari Silva JN, Rudokas M, Bowman TM, Murphy J, Van Hare GF. Coronary sinus morphology in pediatric patients with supraventricular tachycardia. J Interv Card Electrophysiol. 2018;51(2):163-168.

25. Heitkemper M, Sivakumar S, Hatoum H, Dollery J, Lilly SM, Dasi LP. Simple 2-dimensional anatomic model to predict the risk of coronary obstruction during transcatheter aortic valve replacement. J Thorac Cardiovasc Surg. 2020;S0022-5223:30435-9.

\section{Mini Curriculum and Author's Contribution}

1. Uliana Pidvalna - MD; PhD. Contribution: Conceptualization, technical procedure, data acquisition, data interpretation, writing original draft, writing review \& editing, critical review and final approval. ORCID: 0000-0001-7360-8111

2. Lesya Mateshuk-Vatseba - MD; PhD; D.Sc. Contribution: Guiding professor, writing review \& editing, critical review and final approval. ORCID: 0000-0002-3466-5276

3. Dmytro Beshley - MD; MMSc. Contribution: Conceptualization, data interpretation, writing review \& editing, critical review and final approval. ORCID: 0000-0002-4194-517X

4. Kolishetska Marta - MD; PhD; D.Sc. Contribution: Writing review \& editing, critical review and final approval. ORCID: 00000001-9997-0688 